\title{
Synapsin-Promoted Caveolin-1 Overexpression Maintains Mitochondrial Morphology and Function in PSAPP Alzheimer's Disease Mice
}

\author{
Shanshan Wang ${ }^{1,2}\left(\mathbb{D}\right.$, Taiga Ichinomiya ${ }^{1,2,3}$, Yuki Terada ${ }^{1,2,4}$, Dongsheng Wang ${ }^{1,2}$, Hemal H. Patel ${ }^{1,2, *}$ and \\ Brian P. Head 1,2,* \\ 1 Veterans Affairs San Diego Healthcare System, 3350 La Jolla Village Drive, San Diego, CA 92161, USA; \\ shw049@ucsd.edu (S.W.); taiga@nagasaki-u.ac.jp (T.I.); y-1221@naramed-u.ac.jp (Y.T.); \\ dow019@ucsd.edu (D.W.) \\ 2 Department of Anesthesia, University of California San Diego, San Diego, CA 92093, USA \\ 3 Department of Anesthesiology and Intensive Care Medicine, Graduate School of Biomedical Sciences, \\ Nagasaki University, Nagasaki 8528501, Japan \\ 4 Department of Anesthesiology, Nara Medical University, Kashihara 6348521, Japan \\ * Correspondence: hepatel@health.ucsd.edu (H.H.P.); bhead@health.ucsd.edu (B.P.H.)
}

check for updates

Citation: Wang, S.; Ichinomiya, T.; Terada, Y.; Wang, D.; Patel, H.H.; Head, B.P. Synapsin-Promoted Caveolin-1 Overexpression Maintains Mitochondrial Morphology and Function in PSAPP Alzheimer's Disease Mice. Cells 2021, 10, 2487. https://doi.org/10.3390/ cells10092487

Academic Editors: Plamena R. Angelova and Bartosz Szczesny

\section{Received: 4 July 2021}

Accepted: 17 September 2021

Published: 20 September 2021

Publisher's Note: MDPI stays neutral with regard to jurisdictional claims in published maps and institutional affiliations.

Copyright: (c) 2021 by the authors. Licensee MDPI, Basel, Switzerland. This article is an open access article distributed under the terms and conditions of the Creative Commons Attribution (CC BY) license (https:/ / creativecommons.org/licenses/by/ $4.0 /)$.

\begin{abstract}
Mitochondrial dysfunction plays a pivotal role in the Alzheimer's Disease (AD) pathology. Disrupted mitochondrial dynamics (i.e., fusion/fission balance), which are essential for normal mitochondria structure and function, are documented in AD. Caveolin-1 (Cav-1), a membrane/lipid raft (MLR) scaffolding protein regulates metabolic pathways in several different cell types such as hepatocytes and cancer cells. Previously, we have shown decreased expression of Cav-1 in the hippocampus of 9-month (m) old PSAPP mice, while hippocampal overexpression of neurontargeted Cav-1 using the synapsin promoter (i.e., SynCav1) preserved cognitive function, neuronal morphology, and synaptic ultrastructure in 9 and $12 \mathrm{~m}$ PSAPP mice. Considering the central role of energy production in maintaining normal neuronal and synaptic function and survival, the present study reveals that PSAPP mice exhibit disrupted mitochondrial distribution, morphometry, and respiration. In contrast, $S y n C a v 1$ mitigates mitochondrial damage and loss and enhances mitochondrial respiration. Furthermore, by examining mitochondrial dynamics, we found that PSAPP mice showed a significant increase in the phosphorylation of mitochondrial dynamin-related GTPase protein (DRP1), resulting in excessive mitochondria fragmentation and dysfunction. In contrast, hippocampal delivery of SynCav1 significantly decreased p-DRP1 and augmented the level of the mitochondrial fusion protein, mitofusin1 (Mfn1) in PSAPP mice, a molecular event, which may mechanistically explain for the preserved balance of mitochondria fission/fusion and metabolic resilience in $12 \mathrm{~m}$ PSAPP-SynCav1 mice. Our data demonstrate the critical role for Cav-1 in maintaining normal mitochondrial morphology and function through affecting mitochondrial dynamics and explain a molecular and cellular mechanism underlying the previously reported neuroprotective and cognitive preservation induced by SynCav1 in PSAPP mouse model of AD.
\end{abstract}

Keywords: caveolin; gene therapy; mitochondria; oxidative stress; transgenic; Alzheimer's disease

\section{Introduction}

Alzheimer's Disease (AD) is characterized by abundant amyloid- $\beta$ (A $\beta)$ plaques, neurofibrillary tau tangles, mitochondria dysfunction, disrupted synaptic signaling, and loss of synapses, all of which contribute to neuronal degeneration and loss. While the accumulation of damaged mitochondria may result from $\mathrm{A} \beta$ and phosphorylated tau pathology, recent preclinical and clinical studies suggest that mitochondria dysfunction alone can lead to $\mathrm{A} \beta$ oligomeric or fibrillar formation and phosphorylation tau accumulation [1], suggesting that mitochondrial dysfunction may occur earlier and be a more primary cause of AD. Given the high-energy demand of neurons coupled with their complex cellular structure, 
proper mitochondrial dynamics and function are especially important for neurons to allow transport and function of mitochondria at critical sites such as the synapse [2].

Caveolin-1 (Cav-1) is a membrane/lipid raft (MLR), cholesterol-binding, and scaffolding protein that organizes synaptic signaling components, including neurotrophin and neurotransmitter receptors in synaptic membranes [3,4]. However, recent evidence shows that Cav-1 is also involved in metabolic alterations in several different cell types, such as hepatocytes and cancer cells [5-8]. Other isoforms of caveolin (e.g., Cav-3) have been shown to localize to mitochondria and modulate mitochondrial structure and function in stress adaptation $[9,10]$. Similarly, MLRs and Cav-1 have been found to localize in mitochondria in different cell types, including epithelial cells revealed by electron microscopy (EM) [11]. Moreover, in mitotic cells, Cav-1 is upregulated in response to oxidative stress and may serve as a protective mechanism [12-16]. In cancer cells and fibroblasts, oxidative stress stimulates Cav-1 upregulation. This upregulated Cav-1 activates several signaling pathways essential to mitochondrial integrity and function, which in turn mitigates bioenergetic dysfunction [17-19]. As Cav1 is ubiquitously expressed and is regulated by oxidative stress, it remains unclear how Cav-1 expression is impacted in the nervous system.

In neurodegenerative diseases, loss of neuroplasticity is coupled to the downregulation of Cav-1 expression. For example, downregulation of Cav-1 transcript was observed in degenerated neurons in CTE patients [20]. Furthermore, we have also observed decreased Cav-1 in the spinal cord of hSOD1G93A mice (i.e., amyotrophic lateral sclerosis or ALS) and in the hippocampus of PSAPP mice (i.e., AD), while neuron-targeted Cav-1 expression using the synapsin promoter (SynCav1) significantly extended survival and mitochondria count and morphology in the ALS spinal cord [21] and maintained cognitive function, neuronal morphology, and synaptic ultrastructure in PSAPP mice [22]. Mounting evidence suggests an important role played by mitochondria dysfunction in AD. Mitochondria not only provide ATP for axonal transport and neurite growth, synaptic-located mitochondria also provide energy that maintains synaptic function and mobilization of pre-synaptic vesicles, cellular events essential for neurotransmission. While the neuronal membrane phospholipids are composed of mostly polyunsaturated fatty acids, neuron system is particularly vulnerable to oxidative stress. Thus, the dysfunction of mitochondria in neurons can cause synaptic dysfunction and cognitive decline, pathological changes observed in $\mathrm{AD}$ [23]. Considering the pivotal role of oxidative stress and mitochondrial dysfunction to AD neuropathology [24-27], the significant decreased Cav-1 expression, specifically in neural systems, may exacerbate the ability of neurons to adapt to oxidative stress resulting in mitochondrial dysfunction and subsequent neurodegeneration. The possible mechanism of SynCav1-induced neuroprotection in the setting of severe oxidative stress may in part occur through the preservation of mitochondrial morphology. The focus of the current study was to investigate the direct regulatory role of Cav-1 on mitochondrial function and dynamics in PSAPP mice.

\section{Materials and Methods}

\subsection{Animals}

AD-transgenic (Tg) (APPSwePS1d9 a.k.a. PSAPP) and C57BL/6 mice were purchased from Jackson Laboratory (Bar Harbor, ME, USA) and bred inhouse. Transgene negative (WT) littermates were used as controls. Mice were reared (3-5/cage) with free access to food and water. Brain tissue was processed for biochemistry, electron microscopy, and oroboro assay at either $9 \mathrm{~m}$ (early symptomatic) and $12 \mathrm{~m}$ (severely symptomatic).

\subsection{Stereotactic Injection}

Mice ( $3 \mathrm{~m}$ ) were mounted onto a stereotaxic frame under anesthesia ( $2 \%$ isoflurane). Bilateral burr holes were made by a 22-gauge needle. Hippocampal injections using 33gauge, $10 \mu \mathrm{L}$ gas tight syringe (Hamilton, Reno, NV, USA) were controlled by Injectomate (RWD, Shenzhen, China). A total of $1.5 \mu \mathrm{L}$ of adeno-associated virus serotype 9 (AAV9) (viral titer: 109 genome copies (g.c.)/ $\mu \mathrm{L}$ ) containing synapsin-red fluorescent protein 
$(S y n R F P)$ or SynCav1 was injected bilaterally into the hippocampal region over $180 \mathrm{~s}$ at 3 locations (1st site: AP: $1.82 \mathrm{~mm}$, Lat: $1.15 \mathrm{~mm}, \mathrm{DV}: 1.7 \mathrm{~mm}$ : 2nd site: AP: $2.30 \mathrm{~mm}$, Lat: $2.25 \mathrm{~mm}$, DV: $1.75 \mathrm{~mm}$; 3rd site: AP: 2.80, Lat: $2.5 \mathrm{~mm}$, DV: $2.00 \mathrm{~mm}$ ) with $1 \mathrm{~min}$ indwelling time, as previously described $[20,22]$. A representative image of RFP expression was shown in Supplementary Figure S1.

\subsection{Immunoblot Analysis}

Hippocampi were homogenized at $4{ }^{\circ} \mathrm{C}$ in $500 \mathrm{mM}$ sodium carbonate $(\mathrm{pH} 11.0)$ (containing protease and phosphatase inhibitors) followed by sonication $(10 \mathrm{~s} \times 3$ times with $10 \mathrm{~s}$ interval). Lysates were then immunoblotted using primary antibodies against Cav1 (Cell Signaling \#3238; 1:1000), p-DRP (Ser616) (Thermo Fisher; \#PA5-64821, 1:500), DRP1 (cell signaling, \#8570, 1:1000), mitofusion-1 (cell signaling, \#14739, 1:500), mitofusion-2 (cell signaling, \#9482, 1:1000), Tom20 (cell signaling, \#42406, 1:1000), Oxphos (abcam, \#ab110413, 1:2000) and GAPDH (Cell Signaling \#2118s; 1:2000) overnight at $4{ }^{\circ} \mathrm{C}$ followed by incubation with IR-dye labeled secondary antibody for $1 \mathrm{~h}$ at room temperature. Membranes were then visualized by LiCor Odyssey followed by densitometric analysis.

\subsection{Electron Microscopy}

Previously, we observed significant hippocampal synaptic loss and learning deficits in $9 \mathrm{~m}$ PSAPP and stunted dendritic arborization and progressive memory deficits at $12 \mathrm{~m}$ [22]. Thus, for the current study we chose $9 \mathrm{~m}$ and $12 \mathrm{~m}$ PSAPP mice for EM analysis. EM was performed as previously described [28]. Brains were fixed with $2 \%$ glutaraldehyde, treated with $1 \% \mathrm{OsO} 4$, and were then bloc stained with uranyl acetate. For flat embedding of the sections, thin flexible molds were employed to lay the sections as level as possible in LX112 embedding media and then overlayed with plastic coverslips. The flat embedded blocks were visually trimmed to CA1 regions encompassing distal apical dendrites in the "stratum radiatum" of the hippocampus and thin sectioned to $70 \mathrm{~nm}$. Grids were viewed unstained using FEI Tecnai EM scope. Mitochondria located within CA1 were used for analyses. Mitochondria were defined by the presence of cristae and a defined outer membrane [21]. Excitatory synapses were identified by the presence of a prominent, asymmetric post-synaptic density (PSD) [22]. Mitophagy event was defined by the identical autophagosome structure [29,30], the early stage of mitophagy (autophagosome with engulfed mitochondria) can be easily detected through the identification of unique mitochondrial structures such as the cristae (Supplementary Figure S2A). The late stage of mitophagy may be reflected by the single-membrane autolysosomes with residual mitochondria on the basis of their similar electron density with mitochondria (Supplementary Figure S2A). Damaged mitochondria were defined by disorganized cristae, matrix swelling and vacuoles, and abnormal distension between inner and outer mitochondrial membranes. Approximately 5 micrographs at 11,000 magnification (8-12 filed per animal) per animal (4-6 animals per group) were used to perform the analyses. Approximately 150 total mitochondria per group were used in the analyses for area, perimeter and mitochondria count for mitochondria. Micrographs at 9300 magnification (higher magnification contained less mitochondria per micrograph) were used to analyze for the mitochondrial area and count, mitochondrial fusion/fission, and mitophagy event. Mitochondrial size and count were measured using Adobe Photoshop (San Jose, CA, USA) as previously described [21].

\subsection{High-Resolution Mitochondrial Respirometry}

Hippocampal mitochondrial function was measured using an Oroboros O2kRespirometer [31]. Briefly, oxygen polarography was performed at $37^{\circ} \mathrm{C}$, and oxygen flux per tissue mass (pmol O $2 /$ second/mg) was recorded using Datlab software (OROBORO INSTRUMENTS, Innsbruck, Austria). A total of $5 \mathrm{~mL}$ oxygen was injected into each chamber for oxygenation at the beginning of each experiment until oxygen concentration reached $380 \mu \mathrm{M}$. Hippocampal tissue was homogenized in cold Miro5 media on ice containing: $0.5 \mathrm{mM}$ EGTA, $3 \mathrm{mM} \mathrm{MgCl}$, $60 \mathrm{mM}$ K-lactobionate, $20 \mathrm{mM}$ Taurine, $10 \mathrm{mM}$ 
$\mathrm{KH}_{2} \mathrm{PO}_{4}, 20 \mathrm{mM}$ HEPES. $110 \mathrm{mM}$ sucrose and $1.0 \mathrm{~g} / \mathrm{L}$ fatty acid free BSA; $\mathrm{pH}$ 7.1. One milligram of lysate was loaded into each of the $2 \mathrm{~mL}$ respirometers chambers containing $\mathrm{MiRO}_{5}$ and kept at $37^{\circ} \mathrm{C}$ during the measurement. As shown in Figure 1, after 10 min of equilibration, OXPHOS - capacity of complex I (CI OX) linked activity was measured by adding CI-linked substrate pyruvate $(5 \mathrm{mM})$, glutamate $(10 \mathrm{mM})$, malate $(0.5 \mathrm{mM})$, and ADP $(2.5 \mathrm{mM})$; maximum oxidative phosphorylation capacity of CI and II $(\mathrm{CI}+\mathrm{II} \mathrm{OX})$ linked activity was measured by addition of succinate $(10 \mathrm{mM})$, maximum uncoupled capacity (CI + II mUC) were determined following stepwise titration of 2-[2[4-(trifluoromethoxy)phenyl]hydrazinylidene]-propanedinitrile (FCCP). Additionally, CII coupled respiration capacity (CII) was determined in another O2k-Respirometer chamber by addition of $\mathrm{CI}$ inhibitor, rotenone $(0.5 \mu \mathrm{M}), \mathrm{CII}$ substrate succinate $(10 \mathrm{mM})$, and ADP $(2.5 \mathrm{mM})$. Similarly, maximum uncoupled capacity was determined following stepwise titration of 2-[2-[4-(trifluoromethoxy)phenyl]hydrazinylidene]-propanedinitrile (FCCP) (Figure 1B). Oxygen concentration in the chamber was kept at more than $150 \mu \mathrm{M}$ till the end to avoid the limitation of respiration. Duplicate data of each sample were averaged to obtain the final readout. Oxygen flux was expressed as mass-specific respiration (per mg), or as Flux Control Respiration (FCR) by internal normalization for maximum ETS capacity (CI\&II ETS, mUC value) [32]. Mass-specific oxygen consumption rate and FCR was obtained using DatLab 7 software.

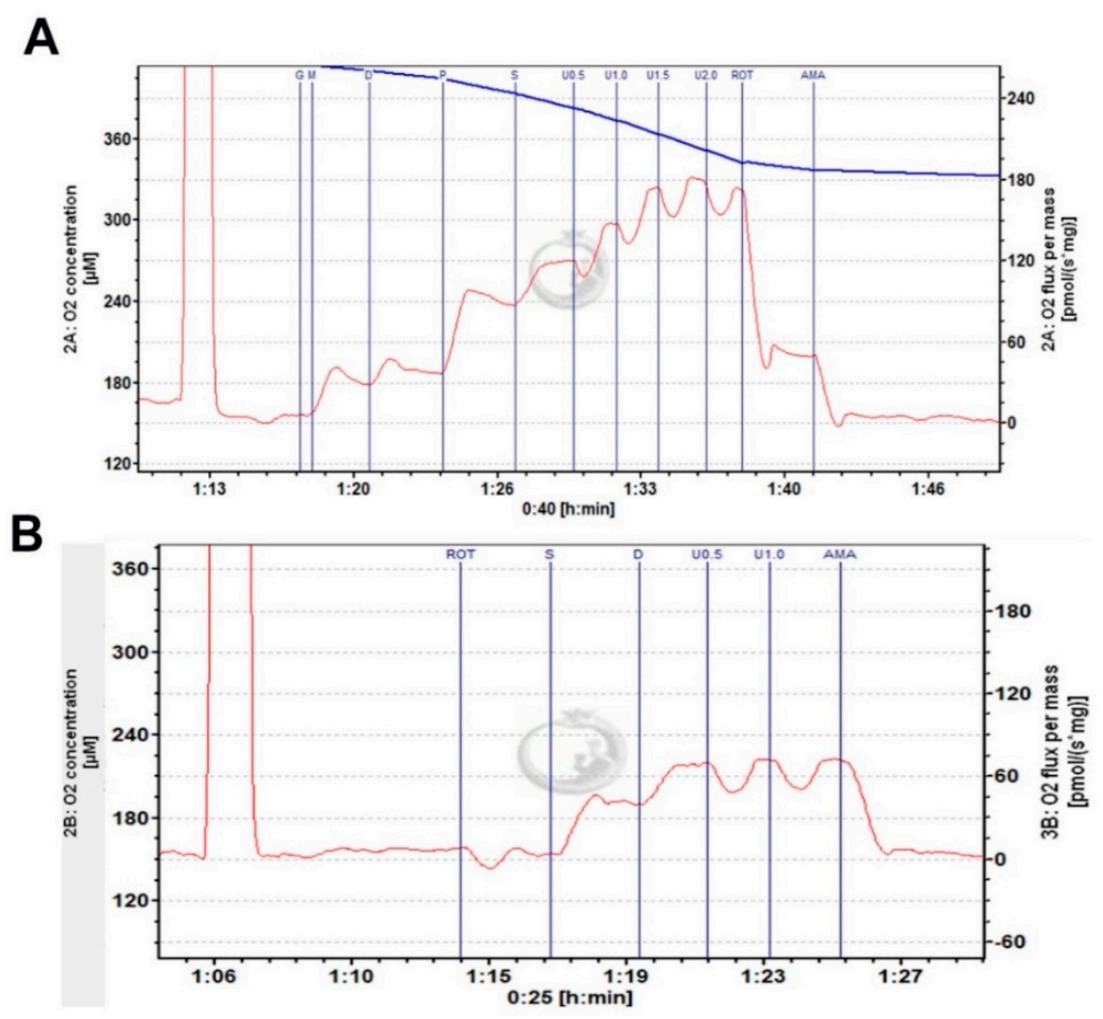

Figure 1. Representative respirometry traces of hippocampal homogenates using the Oroboros O2k-Respirometer. (A) Complex I and II linked activity. (B) Complex II linked activity. Complexes substrates as described in the methods.

\subsection{Statistical Analyses}

Data were checked for normal distribution and analyzed by Student $t$-tests, one-way analysis of variance (ANOVA), or 2-way ANOVA followed by Fisher's LSD multiple comparisons tests as appropriate using GraphPad Prism 7 (La Jolla, California). Data were presented as mean \pm SEM, and significance was assumed when ${ }^{*} p<0.05$. Experimental groups were blinded to the observer, and the code was broken for analysis. 


\section{Results}

3.1. SynCav1 Preserves Soma Mitochondrial Morphology and Dynamics in Hippocampal Pyramidal Neurons of Symptomatic 12 m PSAPP Mice

Mitochondrial morphology alterations and dysfunction have been well documented in AD [33]. Previous work from our group showed that SynCav1 significantly delayed memory deficits in PSAPP mice. We, therefore, performed mitochondrial morphometry using EM as previously described. As shown in Figure 2A, $12 \mathrm{~m}$ PSAPP-SynRFP mice exhibited abnormal mitochondrial morphology consisting of disorganized cristae, matrix swelling and vacuoles, and abnormal distension between inner and outer mitochondrial membranes, morphological indicators of excessive mitochondria damage. Quantification also revealed that $12 \mathrm{~m}$ PSAPP-SynRFP mice exhibited decreased mitochondria number and increased mitochondrial area. In contrast, $12 \mathrm{~m}$ PSAPP-SynCav1 mice exhibited mitochondrial morphology similar to that exhibited by WT-SynRFP mice (Figure 2B,C). Although an increasing trend was observed in mitochondria size in PSAPP-SynRFP group at $9 \mathrm{~m}$, no significant difference was found in total mitochondrial size or count among the 3 groups. However, analysis of abnormal mitochondria confirmed a higher percentage of damaged mitochondria in PSAPP-SynRFP group at $9 \mathrm{~m}$, with no significant difference between PSAPP-SynCav1 and WT-SynRFP (Figure 2D). Mitophagy dysfunction has been observed in several AD mouse models and is recognized as an important contributor to AD pathogenesis [34]. Quantification of mitophagy-like events in both PSAPP-SynRFP and PSAPP-SynCav1 mice were significantly lower compared to WT-SynRFP mice, with no significant difference between the two PSAPP groups (Supplementary Figure S2). These findings demonstrate that $S y n C a v 1$ preserves hippocampal soma mitochondrial profile (i.e., counts and morphology) in PSAPP mice, independent of the mitophagy-related pathway.


Figure 2. Cont. 

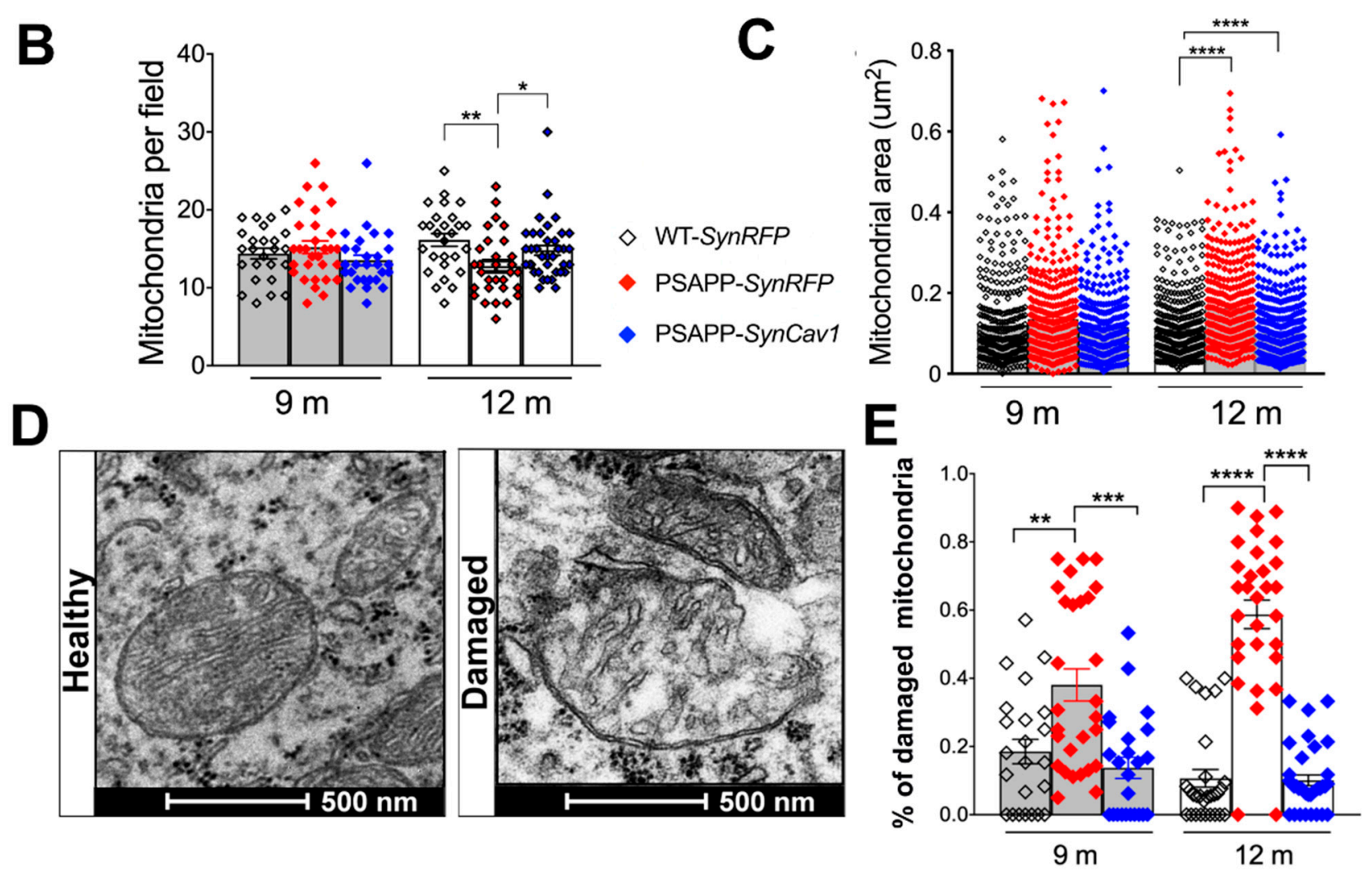

Figure 2. PSAPP-SynRFP mice exhibit less soma mitochondria with larger area, while PSAPP-SynCav1 shows a similar mitochondria profile as WT-SynRFP. (A) EM images of CA1 molecular cell layer consisting of pyramidal cell bodies in $12 \mathrm{~m}$ mice. The red arrow indicates damaged mitochondria with disrupted cristae and empty vacuoles. Quantitation of mitochondrial count and area $(\mathbf{B}, \mathbf{C})$. Representative EM image and quantification of damaged mitochondria $(\mathbf{D}, \mathbf{E})$. Data are presented as mean \pm S.E.M., $n=5$ animals per group with 8-12 micrographs per animal. Data were analyzed using One-Way ANOVA. Significance was assumed when $p<0.05 .{ }^{*} p<0.05,{ }^{* *} p<0.01,{ }^{* * *} p<0.001{ }^{* * * *} p<0.0001$.

\subsection{SynCav1 Preserves Synaptic Mitochondrial Number and Morphology in Hippocampal Pyramidal Neurons of 12 m PSAPP Mice}

Synaptic mitochondria play a vital role in maintaining the synaptic function, including chemical and electrical transmission. Thus, we further analyzed synaptic mitochondria in the stratum radiatum within the hippocampal CA1 subfield, which includes synapses on apical dendrites of pyramidal cells. Although no significant difference was found in total mitochondrial number at $9 \mathrm{~m}$ between groups, quantification revealed a slight increase in mitochondrial area in both PSAPP-SynRFP and PSAPP-SynCav1 mice compared to WT-SynRFP mice. At $12 \mathrm{~m}$, synaptic mitochondrial count in PSAPP-SynRFP mice was significantly decreased while simultaneously exhibiting larger mitochondrial area with no significant difference between PSAPP-SynCav1 and WT-SynRFP, although mitochondrial area from PSAPP-SynCav1 mice was also significantly larger than mitochondria from WT-SynRFP at $12 \mathrm{~m}$. These data suggest that SynCav1 alleviated synaptic mitochondrial damage in PSAPP mice (Figure 3A-D). 




Figure 3. SynCav1 preserves synaptic mitochondrial number and morphology in PSAPP mice. (A) Representative images of synaptic mitochondria in CA1 distal apical dendrites within the stratum radiatum with high magnification of representative region at $12 \mathrm{~m}$. Quantitation of synaptic mitochondrial number and area $(\mathbf{B}, \mathbf{C})$ and \% of total mitochondria relative to the area (D). Representative image and quantification $(\mathbf{E}, \mathbf{F})$ of mitochondria-containing pre-synaptic terminal. Data are presented as mean \pm S.E.M., $n=5-7$ animals per group with 10 micrographs per animal. Data were analyzed using One-Way ANOVA. Significance was assumed when $p<0.05 .{ }^{*} p<0.05,{ }^{* *} p<0.01,{ }^{* * * *} p<0.0001$. 
Previous work from our group already demonstrated PSAPP mice showed decreased total synapses and number of synaptic vesicles (PSVs) at $9 \mathrm{~m} \mathrm{[22].} \mathrm{Because} \mathrm{synaptic}$ mitochondria are essential to synaptic maintenance and function, we further analyzed pre-synaptic mitochondria at both 9 and $12 \mathrm{~m}$. Interestingly, no significant difference was found in the ratio of mitochondria-containing pre-synaptic terminals at $9 \mathrm{~m}$ among the 3 groups, while $12 \mathrm{~m}$ PSAPP-SynRFP mice showed a significantly lower proportion of mitochondrial within pre-synaptic terminals compared to WT-SynRFP mice (Figure 3E,F), findings consistent with others that demonstrated loss of mitochondria in axons and dendrites in postmortem human AD brains [35]. These changes may explain synaptic dysfunction and neuronal damage in AD. In contrast, PSAPP-SynCav1 mice showed a higher proportion of mitochondria within pre-synaptic terminals versus PSAPP-SynRFP, with no significant difference compared to WT-SynRFP mice. These findings indicate that SynCav1 prevents loss of synaptic mitochondria in $12 \mathrm{~m}$ PSAPP mice and may in part explain the cognitive and synaptic preservation previously demonstrated with SynCav1 gene therapy [22].

\subsection{Upregulated P-DRP1 in PSAPP Mice Is Inhibited by SynCav1}

As revealed by mitochondria morphometric analysis, mitochondria morphology among PSAPP groups was found to vary from small, dense mitochondria to extremely large, swollen mitochondria with disrupted cristae. Fission and fusion events are major regulators of mitochondrial morphology. Thus, we further analyzed the ratio of mitochondria that engaged in either fission or fusion as determined by EM (Figure 4A). At $9 \mathrm{~m}$, although there was an increased trend in fusion/fission events in both PSAPP groups, no significant difference was found among the 3 groups. Consistent with these findings, immunoblot analysis showed no significant difference in the expression of mitochondrial fission and fusion proteins in hippocampal tissue between groups at $9 \mathrm{~m}$ (Figure $4 \mathrm{~B}$ ). At $12 \mathrm{~m}$, there was a significant increase of fission/fusion events in PSAPP-SynRFP (Figure 3A) alone with elevated phosphorylated (p-)DRP expression in PSAPP-SynRFP mice compared to WT-SynRFP and PSAPP-SynCav1 mice (Figure 4C,D), results consistent with that observed in postmortem human brains [36]. Interestingly, PSAPP-SynCav1 mice also showed a significant increase in mitofusin-1 (Mfn1) expression compared to other groups, which has been observed to be significantly downregulated in brain tissue from AD patients [37]. Such data suggest that neuronal Cav-1 may dynamically regulate mitochondrial fusion/fission, which is critical to increasing neuroplasticity. 

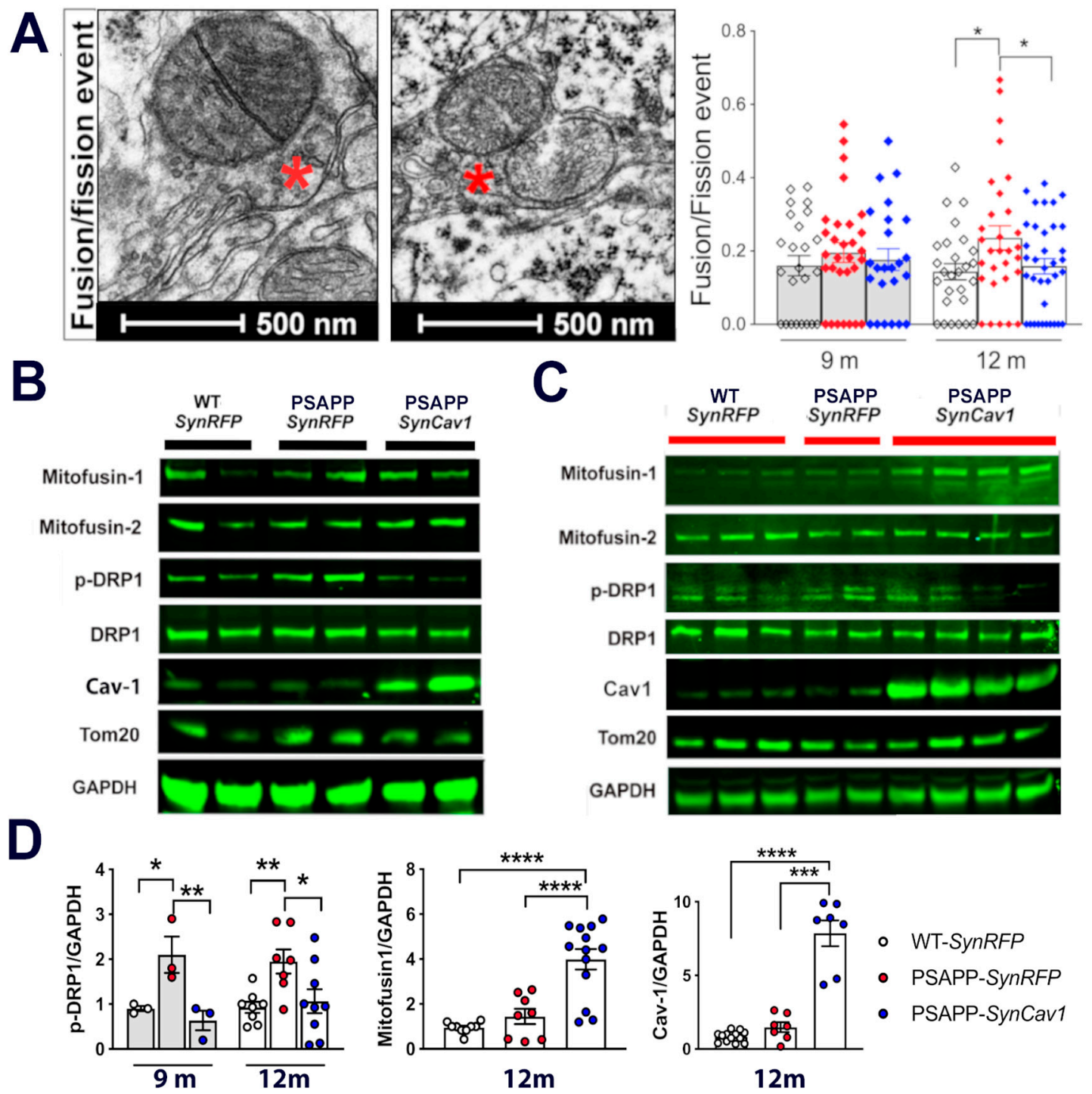

Figure 4. SynCav1 inhibits excessive mitochondria fission observed in $12 \mathrm{~m}$ PSAPP mice hippocampus. (A) Represent image from $9 \mathrm{~m}$. (B) and $12 \mathrm{~m}($ C). (D) Quantification of immunoblots at $12 \mathrm{~m}$. Data are presented as mean \pm S.E.M., $n=3$ per group for $9 \mathrm{~m}, n=6-10$ animals per group for $12 \mathrm{~m}$. Data were analyzed using one-way analysis of variance (ANOVA) followed by Fisher's LSD multiple comparisons test. Significance was assumed when $p<0.05 .{ }^{*} p<0.05$, ${ }^{* *} p$ textless 0.01 , $* * * p<0.001,{ }^{* * * *} p<0.0001$.

\subsection{SynCav1 Augments Hippocampal Mitochondrial Respiration in Symptomatic PSAPP Mice}

Mitochondrial functional plasticity is closely linked to its morphology. Alterations in the fission/fusion machinery, which resulted in abnormal mitochondrial morphology, could significantly affect mitochondrial respiration, which is of particular importance in neurons due to high-energy demands in the brain [24-27]. Since we observed preservation of mitochondrial morphology and dynamics in PSAPP-SynCav1 mice, we, therefore, measured mitochondrial function in hippocampal tissue using the Oroboros O2k-Respirometer. In the presence of complex I and II substrates, no significant difference was found in $9 \mathrm{~m}$ old PSAPP-SynRFP hippocampal homogenates compared to WT-SynRFP (data not shown). A significant decrease in mitochondrial respiration through complex I and II (CI and CII $\mathrm{OX})$, maximum oxidative phosphorylation capacity (CI + II OX), and maximum uncoupled capacity (CI + II mUC) was detected in $12 \mathrm{~m}$ PSAPP-SynRFP mice compared to WT-SynRFP and PSAPP-SynCav1 mice. No significant difference was found between WT-SynRFP and PSAPP-SynCav1 mice (Figure 5A,B). This proportionally increased oxygen consumption 
rate through the whole respiratory state confirmed the mass and quality of mitochondria observed in SynCav1. Since we observed mitochondria content changes across groups by EM, we thus further analyzed oxygen flux control rate (FCR), which can avoid the effect from viability in mitochondrial content [32,38]. When regarding the proportion of ETS used via CI and CII separately, a significant reduction was observed in PSAPP-SynRFP group compared to PSAPP-SynRFP group. On the contrary, PSAPP-SynCav1 group used a significantly higher part of their maximal ETS capacity via CII and a non-significant increase trend in CI (Supplementary Figure S3).
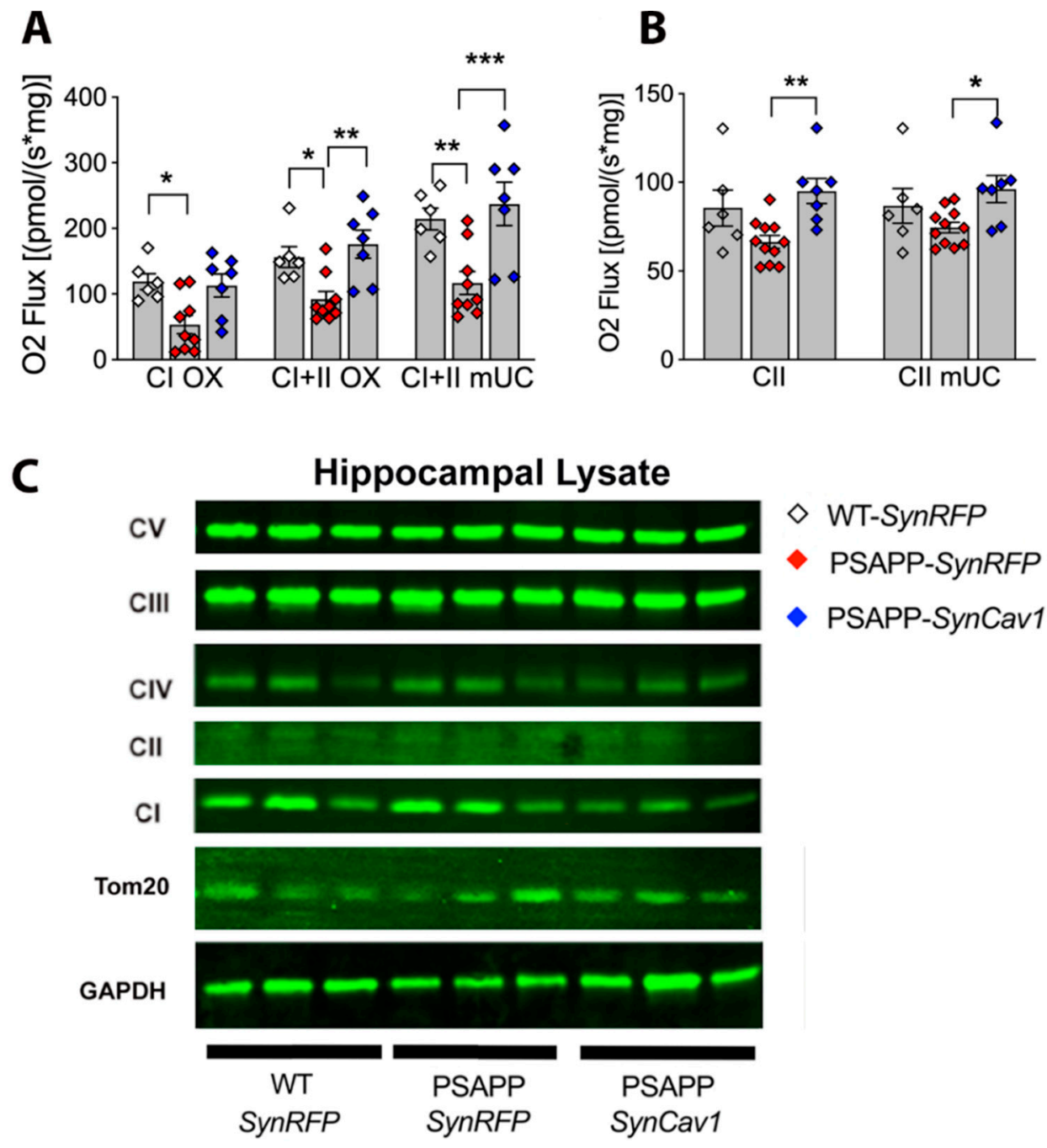

Figure 5. SynCav1 preserves hippocampal mitochondria respiration through complex I and II, maximal oxidative phosphorylation and maximum uncoupled capacity in $12 \mathrm{~m}$ PSAPP mice. (A,B) Quantification of tissue-specific oxygen flux. (C) Presentative blots at $12 \mathrm{~m}$. Data are presented as mean \pm S.E.M., $n=6-10$ per group. Data were analyzed using one-way analysis of variance (ANOVA) followed by Tukey multiple comparisons test. Significance was assumed when $p<0.05$. ${ }^{*} p<0.05,{ }^{* *} p<0.01,{ }^{* * *} p<0.001$.

To better understand the molecular mechanisms underlying these alterations in mitochondrial respiration, we immunoblotted for proteins in the electron transfer chain (ETC) complex at $12 \mathrm{~m}$. The expression levels of different ETC proteins did not significantly change between the 3 groups, as evident by similar expression levels of oxphos complex proteins (Figure 5C). Further immunoblot analysis revealed no significant difference in Tom 20, a mitochondrial import receptor subunit and marker of mitochondrial mass [39], although a non-significant decreased trend was observed in PSAPP-SynRFP group. Such 
data suggest that in lieu of protein deficits in ETC components, the regulatory function of caveolin in AD pathology may be in maintaining mitochondrial function to allowed for coupled respiration.

\section{Discussion}

The pathophysiology of AD is complex, consisting of amyloid deposits and metabolic dysfunction accompanied by hyper-neuroinflammatory responses, all of which exacerbate synaptic loss and neuronal death. Previously, we demonstrated decreased hippocampal Cav-1 in 9 m PSAPP mice, while hippocampal delivery of SynCav1 offered neuroprotection independent of reducing toxic amyloid deposits and astrogliosis [22]. The current study more thoroughly elucidated the neuroprotective mechanism of SynCav1 in PSAPP mice to occur in part through preservation of mitochondria morphology, structural dynamics, and respiratory capacity.

Of all the neuropathological changes observed in $\mathrm{AD}$, the loss of synapse correlates most strongly with cognitive decline. Mitochondria are normally present in areas of high energy demand such as pre-synaptic terminals because synaptic mitochondria provide ATP for synaptic function and mobilization of pre-synaptic vesicles essential for neurotransmission; the spatial distribution of mitochondria in neurons can, therefore, reflect synaptic activity and neuroplasticity. Notably, soma mitochondria still maintain relatively normal morphology and abundance in PSAPP-SynRFP at early symptomatic stage (9 m), in contrast to synaptic mitochondria in PSAPP-SynRFP mice, which already exhibit a larger area compared to WT-SynRFP mice and may explain the early synaptic dysfunction that closely correlated with AD-associated cognitive deficits [40].

The total number and morphology of mitochondria are mainly regulated by the balance of fission and fusion (i.e., mitochondrial dynamics) [41]. While fusion allows for the exchange of lipid membranes and mitochondrial content, fission allows the elimination of irreversibly damaged mitochondria and abnormal content. Mounting evidence reveals that abnormities in mitochondrial dynamics widely precede many of the hallmarks of pathology, which, if appropriately regulated or targeted, could afford significant beneficial effects for patients afflicted with AD. For example, increased p-DRP-1 was observed in several $\mathrm{AD}$ mouse models, resulting in excessive fragmentation of mitochondria and subsequent mitochondrial dysfunction and neuronal damage [37]. In the last few years, several DRP1 inhibitors have shown beneficial effects against mutant protein-induced mitochondrial and synaptic damage in neurodegenerative diseases [42-45]. Here we reveal that SynCav1 inhibits the significant upregulation of p-DRP1 in PSAPP mice. Furthermore, SynCav1-treated PSAPP mice also showed significantly increased Mfn1 expression, another protein that has been targeted for attenuating or reversing axonal degeneration [46]. Mfn1 has previously been shown to be decreased in the same mouse model we used in this study [47]. Furthermore, clinical evidence has revealed that Mfn1 was decreased in AD patients and this decrease correlated with cognitive deficit $[37,48]$. Although we did not observe decreased Mfn1 in our PSAPP group (a possible reason is the time point of sample collection), the consistent elevated Mfn1 in the SynCav1 group may, in part, explain our previously published findings that demonstrated preserved axonal structure and cognitive function in 11-month-old PSAPP mice treated with SynCav1 [22]. These data strongly indicate that the neuroprotective effect from SynCav1 in PSAPP mice is through maintaining mitochondrial dynamics and morphology. Interestingly, although we observed fewer mitochondria in PSAPP mice by electron microscopy, the gross mitochondrial mass measured by Tom20 did not show any significant difference among the groups, suggesting that the gross indicator lacks the biological sensitivity to detect changes in mitochondrial ultrastructure. Several studies have shown that Cav-1 actively participates in maintaining mitochondrial integrity and function by regulating mitochondrial protein quality control [19,49]. Because mitophagy failure has been considered as a driver of AD pathogenesis [34,49-53], we investigated whether the neuroprotective effect of Cav-1 occurred through mitophagic-mediated elimination of damaged mitochondria. However, mitophagy-like events as measured by 
EM failed to show any significant difference between the two PSAPP groups, suggesting that SynCav1 does not affect mitophagy in PSAPP mice (data not shown here).

A growing body of research has highlighted the regulatory role of MLRs and Cav-1 on mitochondrial energetics in non-neuronal systems such as tumors, skeletal, and cardiac systems $[5,10,54,55]$. However, little research has aimed to understand the mechanistic role of Cav-1 in metabolism in the context of neurodegeneration diseases. Here, we demonstrate a clear correlation between Cav-1 expression and mitochondrial morphology and function in neural systems within AD models. Mounting evidence suggests that Abeta accumulation alone can cause mitochondria dysfunction. The decreased Cav-1 contributes to deficits in mitochondrial quality control, which may exacerbate the ability of neurons to adapt to oxidative stress. Conversely, Cav-1 re-introduction with SynCav1 significantly rescued mitochondrial dysfunction. The fact that we did not observe much mitochondrial improvement with SynCav1 in 9-month-old PSAPP mice suggests that neuronal Cav-1 may not affect baseline performance but rather limits the progress of mitochondrial dysfunction at later stages of the disease. These data lend mechanistic insight into the neuroprotective effects of Cav-1 in diseases models of AD. Another potential mechanism through which Cav-1 works may occur through regulating Ca2+ influx, an intracellular event, which has been observed in tumor cells [56], endothelial [57], and smooth muscle cells [58], a process that may couple to mitochondrial resiliency as mitochondria can serve as a calcium sink in cells [59]. Similar effects were observed in a recently published study from Chen and colleagues that showed treadmill exercise protected mitochondrial integrity and inhibited endogenous mitochondrial-mediated apoptosis through Cav-1 upregulation [60]. These results demonstrate a key role in Cav-1 for maintaining mitochondria dynamics and further argue for the overexpression of neuronal Cav-1 as a potential therapeutic strategy to treat neurodegeneration diseases through preservation of mitochondrial function and dynamics.

\section{Conclusions}

In conclusion, this study shows that Cav-1 is a critical regulator of mitochondrial homeostasis in the PSAPP mouse model of AD. Serving as a rapid responder to oxidative stress, Cav-1 has been proven to be essential for proper quality control for maintaining mitochondria homeostasis. Decreased Cav-1 expression in neural systems in PSAPP mice leads to failure of mitochondrial quality control and accumulation of damaged mitochondria, specifically in synaptic regions. Loss of synaptic mitochondrial function invokes severe disturbances in synaptic function, which is preserved with SynCav1 gene transfer. Whether SynCav1 can be exploited as a therapeutic target to treat humans afflicted with $\mathrm{AD}$ needs further investigation.

\section{Patents}

Synapsin-caveolin gene therapy is patented by the U.S. Patent office (US 8,969,077 B2) and owned by the Department of Veterans Affairs (10-084) and UCSD (2010-117-0).

Supplementary Materials: The following are available online at https: / www.mdpi.com/article / 10.3390/cells10092487/s1, Figure S1: Representative image of stereo-microinjection of WT-SynRFP at $12 \mathrm{~m}$ age; Figure S2: Analysis of mitophagy via EM; Figure S3: Quantification of FCR from hippocampal homogenates at 12 months of age.

Author Contributions: S.W. performed experiments, data analyses, and wrote and edited the manuscript; T.I. performed genotyping, colony maintenance; Y.T. performed EM analysis and OROBOROS assay, D.W. performed Immunoblot; H.H.P. assisted with Oroboros analysis and manuscript editing; B.P.H. supervised overall design and edited the manuscript. All authors have read and agreed to the published version of the manuscript.

Funding: Work in the authors' laboratories is supported by Veterans Affairs Merit Award from the Department of Veterans Affairs BX003671 (B.P.H.), and BX005229 (H.H.P.).

Institutional Review Board Statement: All animal protocols were approved by the Veterans Administration San Diego Healthcare System Institutional Animal Care and Use Committee (\#20-030). 
Informed Consent Statement: Not applicable.

Data Availability Statement: Data available upon request from the corresponding authors. Data are not publicly available due to research policies guided by the Department of Veterans Affairs.

Acknowledgments: The authors would like to thank Ying Jones of the UCSD/CMM electron microscopy facility, for TEM sample preparation. The EM facility is supported by NIH equipment grant 1S10OD023527.

Conflicts of Interest: The authors declare no conflict of interest.

\section{References}

1. Rai, S.N.; Singh, C.; Singh, A.; Singh, M.P.; Singh, B.K. Mitochondrial Dysfunction: A Potential Therapeutic Target to Treat Alzheimer's Disease. Mol. Neurobiol. 2020, 57, 3075-3088. [CrossRef]

2. Misgeld, T.; Schwarz, T.L. Mitostasis in Neurons: Maintaining Mitochondria in an Extended Cellular Architecture. Neuron 2017, 96, 651-666. [CrossRef]

3. Mandyam, C.D.; Schilling, J.M.; Cui, W.; Egawa, J.; Niesman, I.R.; Kellerhals, S.E.; Staples, M.C.; Busija, A.R.; Risbrough, V.B.; Posadas, E.; et al. Neuron-Targeted Caveolin-1 Improves Molecular Signaling, Plasticity, and Behavior Dependent on the Hippocampus in Adult and Aged Mice. Biol. Psychiatry 2017, 81, 101-110. [CrossRef]

4. Head, B.P.; Hu, Y.; Finley, J.C.; Saldana, M.D.; Bonds, J.A.; Miyanohara, A.; Niesman, I.R.; Ali, S.S.; Murray, F.; Insel, P.A.; et al. Neuron-targeted caveolin-1 protein enhances signaling and promotes arborization of primary neurons. J. Biol. Chem. 2011, 286, 33310-33321. [CrossRef]

5. Nwosu, Z.C.; Ebert, M.P.; Dooley, S.; Meyer, C. Caveolin-1 in the regulation of cell metabolism: A cancer perspective. Mol. Cancer 2016, 15, 71. [CrossRef] [PubMed]

6. Foster, C.R.; Satomi, S.; Kato, Y.; Patel, H.H. The caveolar-mitochondrial interface: Regulation of cellular metabolism in physiology and pathophysiology. Biochem. Soc. Trans. 2020, 48, 165-177. [CrossRef] [PubMed]

7. Han, M.; Piorońska, W.; Wang, S.; Nwosu, Z.C.; Sticht, C.; Gao, Y.; Ebert, M.P.; Dooley, S.; Meyer, C. Hepatocyte caveolin-1 modulates metabolic gene profiles and functions in non-alcoholic fatty liver disease. Cell Death Dis. 2020, 11, 104. [CrossRef]

8. Han, M.; Nwosu, Z.C.; Piorońska, W.; Ebert, M.P.; Dooley, S.; Meyer, C. Caveolin-1 Impacts on TGF- $\beta$ Regulation of Metabolic Gene Signatures in Hepatocytes. Front. Physiol. 2019, 10, 1606. [CrossRef] [PubMed]

9. Wang, J.; Schilling, J.M.; Niesman, I.R.; Headrick, J.P.; Finley, J.C.; Kwan, E.; Patel, P.M.; Head, B.P.; Roth, D.M.; Yue, Y.; et al. Cardioprotective trafficking of caveolin to mitochondria is Gi-protein dependent. Anesthesiology 2014, 121, 538-548. [CrossRef] [PubMed]

10. Fridolfsson, H.N.; Kawaraguchi, Y.; Ali, S.S.; Panneerselvam, M.; Niesman, I.R.; Finley, J.C.; Kellerhals, S.E.; Migita, M.Y.; Okada, H.; Moreno, A.L.; et al. Mitochondria-localized caveolin in adaptation to cellular stress and injury. FASEB J. 2012, 26, 4637-4649. [CrossRef] [PubMed]

11. Li, W.P.; Liu, P.; Pilcher, B.K.; Anderson, R.G. Cell-specific targeting of caveolin-1 to caveolae, secretory vesicles, cytoplasm or mitochondria. J. Cell Sci. 2001, 114, 1397-1408. [CrossRef]

12. Suchaoin, W.; Chanvorachote, P. Caveolin-1 attenuates hydrogen peroxide-induced oxidative damage to lung carcinoma cells. Anticancer. Res. 2012, 32, 483-490. [PubMed]

13. Pavlides, S.; Tsirigos, A.; Vera, I.; Flomenberg, N.; Frank, P.G.; Casimiro, M.C.; Wang, C.; Fortina, P.; Addya, S.; Pestell, R.G.; et al. Loss of stromal caveolin-1 leads to oxidative stress, mimics hypoxia and drives inflammation in the tumor microenvironment, conferring the "reverse Warburg effect": A transcriptional informatics analysis with validation. Cell Cycle 2010, 9, $2201-2219$. [CrossRef]

14. Wang, S.; Wang, N.; Zheng, Y.; Zhang, J.; Zhang, F.; Wang, Z. Caveolin-1: An Oxidative Stress-Related Target for Cancer Prevention. Oxid. Med. Cell. Longev. 2017, 2017, 7454031. [CrossRef] [PubMed]

15. Martinez-Outschoorn, U.E.; Balliet, R.M.; Rivadeneira, D.B.; Chiavarina, B.; Pavlides, S.; Wang, C.; Whitaker-Menezes, D.; Daumer, K.M.; Lin, Z.; Witkiewicz, A.K.; et al. Oxidative stress in cancer associated fibroblasts drives tumor-stroma co-evolution: A new paradigm for understanding tumor metabolism, the field effect and genomic instability in cancer cells. Cell Cycle 2010, 9, 3256-3276. [CrossRef] [PubMed]

16. Volonte, D.; Galbiati, F. Polymerase I and transcript release factor (PTRF)/cavin-1 is a novel regulator of stress-induced premature senescence. J. Biol. Chem. 2011, 286, 28657-28661. [CrossRef] [PubMed]

17. Dasari, A.; Bartholomew, J.N.; Volonte, D.; Galbiati, F. Oxidative stress induces premature senescence by stimulating caveolin-1 gene transcription through p38 mitogen-activated protein kinase/Sp1-mediated activation of two GC-rich promoter elements. Cancer Res. 2006, 66, 10805-10814. [CrossRef]

18. Powter, E.E.; Coleman, P.R.; Tran, M.H.; Lay, A.J.; Bertolino, P.; Parton, R.G.; Vadas, M.A.; Gamble, J.R. Caveolae control the anti-inflammatory phenotype of senescent endothelial cells. Aging Cell 2015, 14, 102-111. [CrossRef] [PubMed]

19. Volonte, D.; Liu, Z.; Shiva, S.; Galbiati, F. Caveolin-1 controls mitochondrial function through regulation of m-AAA mitochondrial protease. Aging 2016, 8, 2355-2369. [CrossRef] 
20. Mufson, E.J.; He, B.; Ginsberg, S.D.; Carper, B.A.; Bieler, G.S.; Crawford, F.; Alvarez, V.E.; Huber, B.R.; Stein, T.D.; McKee, A.C.; et al. Gene Profiling of Nucleus Basalis Tau Containing Neurons in Chronic Traumatic Encephalopathy: A Chronic Effects of Neurotrauma Consortium Study. J. Neurotrauma 2018, 35, 1260-1271. [CrossRef]

21. Sawada, A.; Wang, S.; Jian, M.; Leem, J.; Wackerbarth, J.; Egawa, J.; Schilling, J.M.; Platoshyn, O.; Zemljic-Harpf, A.; Roth, D.M.; et al. Neuron-targeted caveolin-1 improves neuromuscular function and extends survival in SOD1(G93A) mice. FASEB J. 2019, 33, 7545-7554. [CrossRef] [PubMed]

22. Wang, S.; Leem, J.S.; Podvin, S.; Hook, V.; Kleschevnikov, N.; Savchenko, P.; Dhanani, M.; Zhou, K.; Kelly, I.C.; Zhang, T.; et al. Synapsin-caveolin-1 gene therapy preserves neuronal and synaptic morphology and prevents neurodegeneration in a mouse model of AD. Mol. Ther. Methods Clin. Dev. 2021, 21, 434-450. [CrossRef] [PubMed]

23. Wang, W.; Zhao, F.; Ma, X.; Perry, G.; Zhu, X. Mitochondria dysfunction in the pathogenesis of Alzheimer's disease: Recent advances. Mol. Neurodegener 2020, 15, 30. [CrossRef] [PubMed]

24. Aliev, G.; Palacios, H.H.; Gasimov, E.; Obrenovich, M.E.; Morales, L.; Leszek, J.; Bragin, V.; Solis Herrera, A.; Gokhman, D. Oxidative Stress Induced Mitochondrial Failure and Vascular Hypoperfusion as a Key Initiator for the Development of Alzheimer Disease. Pharmaceuticals 2010, 3, 158-187. [CrossRef] [PubMed]

25. Bonda, D.J.; Wang, X.; Perry, G.; Nunomura, A.; Tabaton, M.; Zhu, X.; Smith, M.A. Oxidative stress in Alzheimer disease: A possibility for prevention. Neuropharmacology 2010, 59, 290-294. [CrossRef] [PubMed]

26. Barber, S.C.; Mead, R.J.; Shaw, P.J. Oxidative stress in ALS: A mechanism of neurodegeneration and a therapeutic target. Biochim. Biophys Acta 2006, 1762, 1051-1067. [CrossRef] [PubMed]

27. Huang, W.J.; Zhang, X.; Chen, W.W. Role of oxidative stress in Alzheimer's disease. Biomed. Rep. 2016, 4, 519-522. [CrossRef]

28. Egawa, J.; Zemljic-Harpf, A.; Mandyam, C.D.; Niesman, I.R.; Lysenko, L.V.; Kleschevnikov, A.M.; Roth, D.M.; Patel, H.H.; Patel, P.M.; Head, B.P. Neuron-Targeted Caveolin-1 Promotes Ultrastructural and Functional Hippocampal Synaptic Plasticity. Cereb. Cortex 2018, 28, 3255-3266. [CrossRef]

29. Zhu, Y.; Chen, G.; Chen, L.; Zhang, W.; Feng, D.; Liu, L.; Chen, Q. Monitoring mitophagy in mammalian cells. Methods Enzym. 2014, 547, 39-55. [CrossRef]

30. Chen, L.; Ma, K.; Han, J.; Chen, Q.; Zhu, Y. Monitoring Mitophagy in Mammalian Cells. Methods Enzym. 2017, 588, 187-208. [CrossRef]

31. Burtscher, J.; Zangrandi, L.; Schwarzer, C.; Gnaiger, E. Differences in mitochondrial function in homogenated samples from healthy and epileptic specific brain tissues revealed by high-resolution respirometry. Mitochondrion 2015, 25, 104-112. [CrossRef]

32. Risiglione, P.; Leggio, L.; Cubisino, S.A.M.; Reina, S.; Paterno, G.; Marchetti, B.; Magri, A.; Iraci, N.; Messina, A. High-Resolution Respirometry Reveals MPP(+) Mitochondrial Toxicity Mechanism in a Cellular Model of Parkinson's Disease. Int. J. Mol. Sci. 2020, 21, 7809. [CrossRef] [PubMed]

33. Albensi, B.C. Dysfunction of mitochondria: Implications for Alzheimer's disease. Int. Rev. Neurobiol. 2019, 145, 13-27. [CrossRef] [PubMed]

34. Chakravorty, A.; Jetto, C.T.; Manjithaya, R. Dysfunctional Mitochondria and Mitophagy as Drivers of Alzheimer's Disease Pathogenesis. Front. Aging Neurosci. 2019, 11, 311. [CrossRef] [PubMed]

35. Pickett, E.K.; Rose, J.; McCrory, C.; McKenzie, C.A.; King, D.; Smith, C.; Gillingwater, T.H.; Henstridge, C.M.; Spires-Jones, T.L. Region-specific depletion of synaptic mitochondria in the brains of patients with Alzheimer's disease. Acta Neuropathol. 2018, 136, 747-757. [CrossRef]

36. Manczak, M.; Calkins, M.J.; Reddy, P.H. Impaired mitochondrial dynamics and abnormal interaction of amyloid beta with mitochondrial protein Drp1 in neurons from patients with Alzheimer's disease: Implications for neuronal damage. Hum. Mol. Genet. 2011, 20, 2495-2509. [CrossRef] [PubMed]

37. Wang, X.; Su, B.; Lee, H.G.; Li, X.; Perry, G.; Smith, M.A.; Zhu, X. Impaired balance of mitochondrial fission and fusion in Alzheimer's disease. J. Neurosci. 2009, 29, 9090-9103. [CrossRef]

38. Gnaiger, E. Capacity of oxidative phosphorylation in human skeletal muscle: New perspectives of mitochondrial physiology. Int. J. Biochem. Cell Biol. 2009, 41, 1837-1845. [CrossRef] [PubMed]

39. Contino, S.; Porporato, P.E.; Bird, M.; Marinangeli, C.; Opsomer, R.; Sonveaux, P.; Bontemps, F.; Dewachter, I.; Octave, J.N.; Bertrand, L.; et al. Presenilin 2-Dependent Maintenance of Mitochondrial Oxidative Capacity and Morphology. Front. Physiol. 2017, 8, 796. [CrossRef] [PubMed]

40. Selkoe, D.J. Alzheimer's disease is a synaptic failure. Science 2002, 298, 789-791. [CrossRef] [PubMed]

41. Wang, X.; Su, B.; Zheng, L.; Perry, G.; Smith, M.A.; Zhu, X. The role of abnormal mitochondrial dynamics in the pathogenesis of Alzheimer's disease. J. Neurochem. 2009, 109 (Suppl. 1), 153-159. [CrossRef]

42. Oliver, D.; Reddy, P.H. Dynamics of Dynamin-Related Protein 1 in Alzheimer's Disease and Other Neurodegenerative Diseases. Cells 2019, 8, 961. [CrossRef]

43. Baek, S.H.; Park, S.J.; Jeong, J.I.; Kim, S.H.; Han, J.; Kyung, J.W.; Baik, S.H.; Choi, Y.; Choi, B.Y.; Park, J.S.; et al. Inhibition of Drp1 Ameliorates Synaptic Depression, Abeta Deposition, and Cognitive Impairment in an Alzheimer's Disease Model. J. Neurosci. 2017, 37, 5099-5110. [CrossRef]

44. Wang, W.; Yin, J.; Ma, X.; Zhao, F.; Siedlak, S.L.; Wang, Z.; Torres, S.; Fujioka, H.; Xu, Y.; Perry, G.; et al. Inhibition of mitochondrial fragmentation protects against Alzheimer's disease in rodent model. Hum. Mol. Genet. 2017, 26, 4118-4131. [CrossRef] [PubMed] 
45. Manczak, M.; Kandimalla, R.; Fry, D.; Sesaki, H.; Reddy, P.H. Protective effects of reduced dynamin-related protein 1 against amyloid beta-induced mitochondrial dysfunction and synaptic damage in Alzheimer's disease. Hum. Mol. Genet. 2016, 25, 5148-5166. [CrossRef] [PubMed]

46. Zhou, Y.; Carmona, S.; Muhammad, A.; Bell, S.; Landeros, J.; Vazquez, M.; Ho, R.; Franco, A.; Lu, B.; Dorn, G.W., 2nd; et al. Restoring mitofusin balance prevents axonal degeneration in a Charcot-Marie-Tooth type 2A model. J. Clin. Invest. 2019, 129, 1756-1771. [CrossRef] [PubMed]

47. Leal, N.S.; Dentoni, G.; Schreiner, B.; Naia, L.; Piras, A.; Graff, C.; Cattaneo, A.; Meli, G.; Hamasaki, M.; Nilsson, P.; et al. Amyloid Beta-Peptide Increases Mitochondria-Endoplasmic Reticulum Contact Altering Mitochondrial Function and Autophagosome Formation in Alzheimer's Disease-Related Models. Cells 2020, 9, 2552. [CrossRef]

48. Yin, J.; Reiman, E.M.; Beach, T.G.; Serrano, G.E.; Sabbagh, M.N.; Nielsen, M.; Caselli, R.J.; Shi, J. Effect of ApoE isoforms on mitochondria in Alzheimer disease. Neurology 2020, 94, e2404-e2411. [CrossRef] [PubMed]

49. Reddy, P.H.; Yin, X.; Manczak, M.; Kumar, S.; Pradeepkiran, J.A.; Vijayan, M.; Reddy, A.P. Mutant APP and amyloid beta-induced defective autophagy, mitophagy, mitochondrial structural and functional changes and synaptic damage in hippocampal neurons from Alzheimer's disease. Hum. Mol. Genet. 2018, 27, 2502-2516. [CrossRef] [PubMed]

50. Martín-Maestro, P.; Gargini, R.; García, E.; Simón, D.; Avila, J.; García-Escudero, V. Mitophagy Failure in APP and Tau Overexpression Model of Alzheimer's Disease. J. Alzheimer's Dis. 2019, 70, 525-540. [CrossRef]

51. Cummins, N.; Tweedie, A.; Zuryn, S.; Bertran-Gonzalez, J.; Götz, J. Disease-associated tau impairs mitophagy by inhibiting Parkin translocation to mitochondria. EMBO J. 2019, 38, e99360. [CrossRef] [PubMed]

52. Cai, Q.; Jeong, Y.Y. Mitophagy in Alzheimer's Disease and Other Age-Related Neurodegenerative Diseases. Cells 2020, 9 , 150. [CrossRef]

53. Fang, E.F.; Hou, Y.; Palikaras, K.; Adriaanse, B.A.; Kerr, J.S.; Yang, B.; Lautrup, S.; Hasan-Olive, M.M.; Caponio, D.; Dan, X.; et al. Mitophagy inhibits amyloid- $\beta$ and tau pathology and reverses cognitive deficits in models of Alzheimer's disease. Nat. Neurosci. 2019, 22, 401-412. [CrossRef]

54. Niesman, I.R.; Zemke, N.; Fridolfsson, H.N.; Haushalter, K.J.; Levy, K.; Grove, A.; Schnoor, R.; Finley, J.C.; Patel, P.M.; Roth, D.M.; et al. Caveolin isoform switching as a molecular, structural, and metabolic regulator of microglia. Mol. Cell Neurosci. 2013, 56, 283-297. [CrossRef]

55. Shah, D.S.; Nisr, R.B.; Stretton, C.; Krasteva-Christ, G.; Hundal, H.S. Caveolin-3 deficiency associated with the dystrophy P104L mutation impairs skeletal muscle mitochondrial form and function. J. Cachexia Sarcopenia Muscle 2020, 11, 838-858. [CrossRef]

56. Rimessi, A.; Marchi, S.; Patergnani, S.; Pinton, P. H-Ras-driven tumoral maintenance is sustained through caveolin-1-dependent alterations in calcium signaling. Oncogene 2014, 33, 2329-2340. [CrossRef] [PubMed]

57. Murata, T.; Lin, M.I.; Stan, R.V.; Bauer, P.M.; Yu, J.; Sessa, W.C. Genetic evidence supporting caveolae microdomain regulation of calcium entry in endothelial cells. J. Biol. Chem. 2007, 282, 16631-16643. [CrossRef]

58. Adebiyi, A.; Narayanan, D.; Jaggar, J.H. Caveolin-1 assembles type 1 inositol 1,4,5-trisphosphate receptors and canonical transient receptor potential 3 channels into a functional signaling complex in arterial smooth muscle cells. J. Biol. Chem. 2011, 286, 4341-4348. [CrossRef] [PubMed]

59. Celsi, F.; Pizzo, P.; Brini, M.; Leo, S.; Fotino, C.; Pinton, P.; Rizzuto, R. Mitochondria, calcium and cell death: A deadly triad in neurodegeneration. Biochim. Biophys. Acta 2009, 1787, 335-344. [CrossRef] [PubMed]

60. Pan, G.; Zhang, H.; Zhu, A.; Lin, Y.; Zhang, L.; Ye, B.; Cheng, J.; Shen, W.; Jin, L.; Liu, C.; et al. Treadmill exercise attenuates cerebral ischaemic injury in rats by protecting mitochondrial function via enhancement of caveolin-1. Life Sci. 2021, 264, 118634. [CrossRef] [PubMed] 